\title{
Clinical significance of enlarged lateral pelvic lymph nodes before and after preoperative chemoradiotherapy for rectal cancer
}

\author{
YASUHIRO INOUE, SUSUMU SAIGUSA, JUNICHIRO HIRO, YUJI TOIYAMA, \\ TOSHIMITSU ARAKI, KOJI TANAKA, YAUSHIKO MOHRI and MASATO KUSUNOKI
}

\begin{abstract}
Division of Reparative Medicine, Department of Gastrointestinal and Pediatric Surgery, Institute of Life Sciences, Mie University Graduate School of Medicine, Tsu, Mie 514-8507, Japan
\end{abstract}

Received August 25, 2015; Accepted March 16, 2016

DOI: $10.3892 / \mathrm{mco} .2016 .855$

\begin{abstract}
Preoperative chemoradiotherapy (CRT) with total mesorectal excision (TME) is the widely accepted treatment for rectal cancer (RC) in Western countries. However, there remains controversy as to whether preoperative CRT is useful in tumors that extend beyond the mesorectum, including metastasis to the lateral pelvic lymph nodes (LPLN). The aim of this study was to assess the prognostic significance of LPLN enlargement in patients with $\mathrm{RC}$ who receive preoperative CRT followed by TME without LPLN dissection. We evaluated the prognostic effect of radiographic LPLN enlargement before and after CRT, as well as the patients' clinicopathological and genetic profiles. Of the 104 patients investigated, pretreatment imaging identified $19(18 \%)$ as LPLN-positive (>7 mm in diameter). Of these 19 patients, 7 (37\%) exhibited LPLN downsizing to $<7 \mathrm{~mm}$ following CRT. The median follow-up period was 52 months. The 5 -year cancer-specific survival (CSS) or relapse-free survival (RFS) did not differ significantly between patients who did and those who did not have positive LPLN on pretreatment imaging. However, LPLN that remained positive after CRT were significantly associated with poorer 5-year CSS ( 73 vs. $84 \%$, respectively; $\mathrm{P}=0.0052$ ) and RFS (32 vs. $78 \%$, respectively; $\mathrm{P}=0.0264$ ). None of the patients whose LPLN were downsized to $<7 \mathrm{~mm}$ following CRT developed recurrence; however, those with positive LPLN after CRT had a $55 \%$ higher recurrence rate, characterized by delayed local recurrence, a pattern that may be affected by certain chemokines. In conclusion, changes in initially positive LPLN ( $>7 \mathrm{~mm}$ ) may predict the prognosis of patients with RC who receive preoperative CRT-TME. LPLN positivity after CRT was associated with shorter CSS and RFS. Strategies to
\end{abstract}

Correspondence to: Dr Yasuhiro Inoue, Division of Reparative Medicine, Department of Gastrointestinal and Pediatric Surgery, Institute of Life Sciences, Mie University Graduate School of Medicine, 2-174 Edobashi, Tsu, Mie 514-8507, Japan

E-mail: yasinoue@clin.medic.mie-u.ac.jp

Key words: rectal cancer, lateral pelvic lymph nodes, preoperative chemoradiotherapy improve patient survival may include selective LPLN dissection or more aggressive multimodality therapy.

\section{Introduction}

Recent advances in the treatment of rectal cancer (RC) have reduced local recurrence rates and improved overall survival. Preoperative chemoradiotherapy (CRT) with total mesorectal excision (TME) is the widely accepted treatment for RC in Western countries. However, different treatment strategies have been developed in Japan and Western countries to achieve postoperative local control for advanced RC, particularly for tumors that extend beyond the mesorectum, including metastasis to the lateral pelvic lymph nodes (LPLN). LPLN dissection is often performed with TME in advanced low RC in Japan, as TME does not remove LPLN, which may contain metastasis and lead to local recurrence. The incidence of LPLN metastasis is reportedly $10-20 \%$ in patients with advanced low RC who undergo LPLN dissection (1-5). Although a retrospective analysis has suggested that LPLN dissection is equivalent to preoperative CRT in terms of local control for patients with lower RC (6), the effect of preoperative CRT on patients with involved LPLN has not been fully investigated. Recent reports have demonstrated that pretreatment imaging of RC patients using computed tomography (CT) and/or magnetic resonance imaging (MRI) may detect LPLN involvement with a high degree of accuracy (7). Pretreatment imaging of LPLN in RC patients who undergo preoperative CRT reportedly has some clinical significance. Dharmarajan et al evaluated the outcomes of patients whose LPLN enlargement was identified with pretreatment imaging and who were treated with preoperative CRT-TME without LPLN dissection (8), and concluded that clinically enlarged LPLN do not affect prognosis following preoperative CRT-TME in stage III RC patients. However, Akiyoshi et al reported that the incidence of LPLN metastasis is high even after preoperative CRT, and selective LPLN dissection in patients with suspected LPLN metastasis based on pretreatment imaging may improve local disease control and patient survival (9).

Although enlarged LPLN response to preoperative CRT may help guide the selection of lymphadenectomy or active adjuvant chemotherapy for persistently enlarged LPLN, the clinical significance of this response is unclear. The aim of this study was to determine the outcomes of patients whose LPLN 
were enlarged on pretreatment imaging and who received preoperative CRT-TME without LPLN dissection. We also sought to determine the characteristics of enlarged LPLN following preoperative CRT, and the prognostic significance of enlarged LPLN response to preoperative CRT.

\section{Patients and methods}

Patients. Prospectively maintained data of all the patients $(\mathrm{n}=104)$ who underwent preoperative CRT for advanced, biopsy-proven RC at the Department of Gastrointestinal Surgery, Mie University Hospital (Tsu, Japan), from January, 2001 to July, 2013 were retrospectively evaluated. The criteria for preoperative CRT were as follows: Patients aged 20-80 years who had cT2-4 or $\mathrm{cN}+$ disease, with an Eastern Cooperative Oncology Group performance status of 0 or 1. Our Institutional Ethics Committee approved the study protocol, and written informed consent was obtained from all the patients who entered the study.

Clinical staging and LPLN evaluation. Pretreatment clinical stage was assessed based on multidetector CT (MDCT) and/or MRI. Tumor location was determined by endoscopy, barium enema and transrectal ultrasound, and LN enlargement was determined by MDCT and/or MRI. All the images were retrospectively reviewed by one colorectal surgeon and two experienced radiologists, who were blinded to the precise clinical information. The maximum long and short axis diameter of the LPLNs were measured before and after the preoperative CRT (i.e., just before rectal surgery), and LPLN positivity was defined as any lymph node sized $>7 \mathrm{~mm}$ in the long-axis diameter in the lateral pelvic area, according to previous reports $(9,10)$. Reduction of LPLN size following preoperative CRT was defined as initially positive LPLN downsized to $<7 \mathrm{~mm}$.

CRT schedules and surgery. Patients with RC were treated with short-course (20 Gy in four fractions, $\mathrm{n}=58$ ) or long-course (45-50 Gy in 25 fractions, $n=46$ ) external irradiation using the 4-field approach. A CT-based treatment planning system was mandatory for defining the planning target volume, including the primary tumor, the surrounding mesorectum and bilateral LPLN, including the internal iliac, obturator, external iliac and common iliac areas, according to the Japanese Society for Cancer of the Colon and Rectum (JSCCR) classification (11). All the patients underwent concurrent 5-fluorouracil (5-FU)-based chemotherapy, including 5-FU/leucovorin, tegafur/uracil and S-1 $(12,13)$. Long-course CRT was mainly used for patients with low-lying tumors and intended sphincter preservation, or tumors close to the circumferential margin. The mean interval between the completion of CRT and TME was 10 days in short-course and 6-8 weeks in long-course CRT. LPLN dissection was not introduced in this study, with the exception of staging biopsy in 1 patient.

Clinical response and tumor regression after CRT. The degree of histopathological tumor regression was defined based on the Guidelines for the Clinical and Pathological Studies on Carcinoma of the Colorectum, and was classified into 4 grades: Grade 0 , no necrosis or regressive change; grade 1a, 66\% vital residual tumor cells (VRTCs); grade $1 \mathrm{~b}$, $\sim 33-66 \%$ VRTCs; grade 2, <33\% VRTCs; and grade 3, no VRTCs (11). Non-responders were defined as patients with histopathological tumor regression grades $0-1 \mathrm{~b}$, and responders as those with grades 2-3.

Molecular analysis of resected specimens. Formalin-fixed paraffin-embedded specimens following CRT were available according to our previous study (14). Quantitative reverse transcription-polymerase chain reaction (RT-PCR) analysis was performed with the SYBR Green PCR Master Mix (Applied Biosystems, Foster City, CA) using the Applied Biosystems 7500 Real-Time PCR system. Previously reported genes associated with the CRT effects were evaluated in this study (14). The primers for cyclin-dependent kinase inhibitor 1A [CDKN1A $\left.\left(p 21^{C i p}\right)\right]$, vascular endothelial growth factor $(V E G F)$, hypoxia-inducible factor 1 (HIFl), hepatocyte growth factor $(H G F), C D 133$, sex determining region Y-box 2 (SOX2), octamer-binding transcription factor 4 (OCT4), BCL2-associated X protein $(B A X)$, B-cell lymphoma 2 (BCL2), C-X-C motif chemokine 12 (CXCL12), C-X-C chemokine receptor type 4 (CXCR4), and $\beta$-actin genes were designed with Primer3 software (Biology Workbench version 3.2, San Diego Supercomputer Center, University of California, San Diego, CA, USA). The primer sequences are listed in Table I. Immunohistochemistry for CXCL12 was also performed in 1 patient who underwent LPL sampling following local recurrence, according to our previous study (15). Monoclonal anti-human CXCL12 antibody (clone 79018; dilution 1:100; cat. no. MAB350; R\&D systems, Minneapolis, MN, USA) was used to implement the labeled streptavidin-biotin method (LASB2 kit/HRP, DakoCytomation, Denmark). The tumors were classified as CXCL12-negative if all or most of the cancer cells were unstained $(<10 \%$ positive cells) and as CXCL12-positive if $>10 \%$ of the cells were immunostained.

Statistical analysis. JMP version 7 software (SAS Institute, Cary, NC, USA) was used for statistical analyses. The data are presented as mean \pm standard error. Contingency tables were analyzed using Fisher's exact test or the $\chi^{2}$ test with Yates's correction. Correlations between continuous and categorical variables were evaluated by the Mann-Whitney U test. Survival curves were constructed according to the Kaplan-Meier method and differences were analyzed using the log-rank test. Each significant predictor identified was assessed by multivariate analysis using the Cox's proportional hazards model. $\mathrm{P}<0.05$ was considered to indicate statistically significant differences.

\section{Results}

Patient characteristics. Of the 104 patients, 19 (18.3\%) had positive LPLN $(>7 \mathrm{~mm}$ ) identified on pretreatment imaging and $85(81.7 \%)$ were LPLN-negative. A comparison of demographic characteristics and treatment details between the LPLN-positive and -negative cases is shown in Table II. The mean distance of the tumors from the anal verge was not significantly different between the two groups. However, cT4 was more common among LPLN-positive (52.6\%) than LPLN-negative cases (12.9\%); thus, abdominoperineal rectal resection was more frequently applied in the LPLN-positive group. The pretreatment serum carcinoembryonic antigen (CEA) level was significantly higher 
Table I. Primer sequences of target genes.

\begin{tabular}{lll}
\hline Gene symbol & \multicolumn{1}{c}{ Forward } & \multicolumn{1}{c}{ Reverse } \\
\hline$C D K N 1 A\left(p 21^{C i p l}\right)$ & GACTCTCAGGGTCGAAAAACG & GGATTAGGGCTTCCTCTTGG \\
$V E G F$ & CAGAAGGAGGAGGGCAGAA & CTCGATTGGAGGCAGTAGC \\
$H I F 1$ & CCGCTGGAGACACAATCATA & CTTCCTCAAGTTGCTTTTCA \\
$H G F$ & ATTTGGCCATGTTTTGACC & AGCTGCGTCCTTTACCAATG \\
$C D 133$ & GCTTTGCAATCTCCCTGTTG & AGCTGCGTCCTTTACCAATG \\
SOX2 & CAAGATGCACAACTCGGAGA & GCTTAGCCTCGTCGATGAAC \\
OCT4 & CTGGAGAAGGAGAAGCTGGA & CAAATTGCTCGAGTTCTTCTG \\
$B C L 2$ & CTTTGCCAGCAAACTGGTG & CAGCCCATGATGGTTCTGA \\
$C X C L 12$ & TCGCCCTGTGGATGACTGA & CAGACAGAGCCAGGTTCTGA \\
$C X C R 4$ & ATGAACGCCAAGGTCGTG & ACATGGCTTTCGAAGAATCG \\
ACTB & CAGCAGGTAGCAAAGTGACG & ATAGTCCCCTGACCCTTT \\
\hline
\end{tabular}

$C D K N 1 A\left(p 21^{C i p l}\right)$, cyclin-dependent kinase inhibitor 1A; VEGF, vascular endothelial growth factor; HIF 1, hypoxia-inducible factor 1; $H G F$, hepatocyte growth factor; $S O X 2$, sex-determining region Y-box 2; OCT4, octamer-binding transcription factor; BAX, BCL2-associated X protein; BCL2, B-cell lymphoma 2; CXCL12, C-X-C motif chemokine 12; CXCR4, C-X-C chemokine receptor type 4; ACTB, $\beta$-actin.

in the LPLN-positive compared with the LPLN-negative group (63.1 vs. 13.3, respectively; $\mathrm{P}=0.005)$, although the two groups did not significantly differ in terms of age, gender, or histological type. The proportion of short- course and long-course CRT also did not differ significantly between the two groups.

Radiation effects on LPLN-positive status. After preoperative CRT, 7 of the $19(36.8 \%)$ patients with positive LPLN had downsized to $<7 \mathrm{~mm}$. The maximum diameter of initially positive LPLN (mean, $15.1 \mathrm{~mm}$; range, $8-45 \mathrm{~mm}$ ) was significantly reduced after preoperative CRT (mean, $10.9 \mathrm{~mm}$; range, $0-45 \mathrm{~mm} ; \mathrm{P}=0.009$ ). Rectal resection was performed in 103 patients, and grade $\geq 2$ response was confirmed in $38(36.5 \%)$ patients. These primary tumor responders were not correlated with LPLN-positive status before and after CRT, but were correlated with a lower rate of pathological mesorectal lymph node (MLN) metastasis (10.5 vs. 47.0\%; $\mathrm{P}=0.0002)$. Although the CRT effect were not significantly correlated with LPLN-positive status and primary tumor, the LPLN-positive group had significantly more cases of pathological MLN metastasis compared with the LPLN-negative group (2.4 vs. 0.8, respectively; $\mathrm{P}=0.008)$. The patient and tumor characteristics according to LPLN-positive status after preoperative CRT are shown in Table III. More MLN metastases were found in the LPLN-positive group after preoperative CRT (3.7 vs. 0.7; $\mathrm{P}<0.0001$ ) and the $\mathrm{LN}$ ratio (positive/harvested $\mathrm{LNs}$ ) in LPLN-positive patients after CRT $(n=12)$ was significantly higher compared with that in LPLN-negative patients after CRT, although 5 of the $12(41.2 \%)$ patients had no pathological MLN metastasis.

Oncological outcomes. The median follow-up period was 52.2 months (range, 5.7-154.3 months). Of the 103 patients who underwent primary resection, recurrence developed in 27 patients, including local recurrence in 7 (6.8\%) and distant recurrence in $20(19.4 \%)$ patients. The 5-year cancer-specific survival (CSS) and relapse-free survival (RFS) did not differ significantly between patients with and without positive LPLN on pretreatment imaging (82.6 vs. $83.4 \%$ and 75.2 vs. $56.6 \%$, respectively). However, LPLN-positive status after CRT was significantly associated with poor 5-year CSS (72.9 vs. 84.8\%; $\mathrm{P}=0.005$ ) and RFS (32.4 vs. 77.1\%; $\mathrm{P}=0.04$ ) (Fig. 1). For the entire cohort, the 3-year cumulative local recurrence rates were $12.5 \%$ in the LPLN-positive and $2.8 \%$ in the LPLN-negative groups after CRT. The univariate analysis identified cT4, LPLN-positive status after CRT, pMLN metastasis and high serum CEA level as significant predictors of poor CSS. The multivariate analysis demonstrated that pMLN metastasis and cT4, but not enlarged LPLN, were independent prognostic factors for CSS [odds ratio $(\mathrm{OR})=3.72,95 \%$ confidence interval $(\mathrm{CI}): 1.34-10.31, \mathrm{P}=0.01$; and $\mathrm{OR}=3.65,95 \% \mathrm{CI}: 1.28-10.42, \mathrm{P}=0.02$, respectively] in patients with RC who received preoperative CRT (Table IV). Also, CRM positivity, cT4 and pMLN metastasis were significant predictors of RFS in the univariate analysis, and pMLN metastasis was an independent prognostic factor for RFS in the multivariate analysis $(\mathrm{OR}=4.61,95 \% \mathrm{CI}$ : 2.00-10.53, $\mathrm{P}=0.0003)$ (Table IV). However, the Kaplan-Meier survival analysis revealed significant differences in CSS among the three groups (pN0, pMLN metastasis and LPLN-positive groups) after CRT $(\mathrm{P}=0.003)$ (Fig. 2). Interestingly, survival differences between pMLN metastasis and LPLN-positive cases after CRT were confirmed $>5$ years after surgery, due to delayed recurrence. All 7 patients who exhibited LPLN downsizing to $<7 \mathrm{~mm}$ after CRT had no recurrence. However, of the 11 patients whose LPLN remained positive after CRT and underwent surgery, 6 (54.5\%) developed recurrence. Local recurrence developed in 4 patients at a median of 47 months (range, 23-59 months), whereas distant recurrence developed in 2 patients at a median of 39 months (range, 11-60 months). The sites of local recurrence were 1 to the anterior pelvis at 48 months, 2 to the posterior pelvis at 38.8 and 45.3 months, and 1 LPLN at 58.7 months after surgery.

Molecular characteristics of RC according to the CRT effect on LPLN-positive status. To evaluate the molecular 
Table II. Patient and tumor characteristics according to enlarged LPLN identified on pretreatment imaging.

\begin{tabular}{|c|c|c|c|c|}
\hline Characteristics & All patients & LPLN-positive & LPLN-negative & P-value \\
\hline Patients, $\mathrm{n}$ & 104 & 19 & 85 & \\
\hline Age (mean, years) & 62.4 & 61.8 & 62.5 & 0.78 \\
\hline Gender (male/female) & $77 / 27$ & $16 / 3$ & $61 / 24$ & 0.26 \\
\hline Tumor distance (mean, $\mathrm{cm}$ ) & 3.9 & 3.6 & 4.0 & 0.52 \\
\hline \multicolumn{5}{|l|}{ Clinical UICC stage } \\
\hline I/II/III/IV & $11 / 13 / 79 / 1$ & $0 / 0 / 19 / 0$ & $11 / 13 / 60 / 1$ & 0.06 \\
\hline cT4 $(\%)$ & $21(20.2)$ & $10(52.6)$ & $11(12.9)$ & $<0.0001$ \\
\hline $\mathrm{cN}$ metastasis $(\%)$ & $80(76.9)$ & $19(100)$ & $61(71.8)$ & 0.008 \\
\hline Histological differentiation & & & & 0.10 \\
\hline High/moderate & 93 & 15 & 78 & \\
\hline Mucinous/poor & 11 & 4 & 7 & \\
\hline Pretreatment CEA $(n g / \mu 1)$ & 22.4 & 63.1 & 13.3 & 0.005 \\
\hline \multicolumn{5}{|l|}{ CRT schedule } \\
\hline Short-course/long-course & $58 / 46$ & $10 / 9$ & $48 / 37$ & 0.47 \\
\hline \multicolumn{5}{|l|}{ Adjuvant chemotherapy } \\
\hline Yes $(\%)$ & $97(93.3)$ & $18(94.7)$ & $78(91.8)$ & 0.35 \\
\hline \multicolumn{5}{|l|}{ Surgery } \\
\hline Yes $(\%)$ & $103(99.0)$ & $18(94.7)$ & $85(100)$ & 0.18 \\
\hline Surgical procedure & & & & 0.0001 \\
\hline LAR & 28 & 2 & 26 & \\
\hline CAA & 63 & 9 & 54 & \\
\hline APR & 7 & 5 & 2 & \\
\hline Hartmann's procedure & 2 & 2 & 0 & \\
\hline Local excision & 3 & 0 & 3 & \\
\hline Intersphincter resection $(\%)$ & $43(41.7)$ & $7(36.8)$ & $36(42.4)$ & 0.66 \\
\hline $\operatorname{APR}(\%)$ & $7(6.7)$ & $5(26.3)$ & $2(2.4)$ & 0.0002 \\
\hline \multicolumn{5}{|l|}{ Pathological UICC stage } \\
\hline 0/I/II/III/IV & $7 / 24 / 36 / 35 / 2$ & $0 / 2 / 9 / 8 / 0$ & $7 / 22 / 27 / 27 / 2$ & 0.27 \\
\hline pN metastasis $(\%)$ & $35(33.7)$ & $8(42.1)$ & $27(31.8)$ & 0.39 \\
\hline CRM positivity (\%) & $3(2.9)$ & $1(5.6)$ & $2(2.4)$ & 0.46 \\
\hline \multicolumn{5}{|l|}{ Pathological effects } \\
\hline Grade $0 / 1 \mathrm{a} / 1 \mathrm{~b} / 2 / 3$ & $1 / 36 / 29 / 31 / 7$ & $1 / 5 / 5 / 7 / 1$ & $0 / 31 / 24 / 24 / 6$ & 0.25 \\
\hline Grade $\geq 2(\%)$ & $38(36.5)$ & $8(42.1)$ & $30(35.3)$ & 0.58 \\
\hline $\mathrm{pCR}(\%)$ & $7(6.7)$ & $1(5.2)$ & $6(7.0)$ & 0.78 \\
\hline Mesorectal LN harvest & 8.5 & 9.9 & 8.2 & 0.25 \\
\hline Positive LNs (mean) & 1.1 & 2.4 & 0.8 & 0.008 \\
\hline Negative LNs (mean) & 7.4 & 7.4 & 7.4 & 0.97 \\
\hline LN ratio (postitive/harvested) & 0.12 & 0.19 & 0.1 & 0.19 \\
\hline $\begin{array}{l}\text { Interval between CRT and surgery } \\
\text { (days), mean }\end{array}$ & 35.6 & 45.6 & 33.8 & 0.20 \\
\hline
\end{tabular}

Bold print indicates statistical significance. LPLN, lateral pelvic lymph nodes; UICC, Union for International Cancer Control; CRM, circumferential resection margin; CEA, carcinoembryonic antigen; CRT, chemoradiotherapy; LAR, low anterior resection; CAA, colo-anal anastomosis; APR, abdominoperineal rectal resection; pCR, pathological complete response.

characteristics of RC with LPLN-positive status even after CRT, the expression of genes reportedly associated with CRT were measured using RT-PCR. We observed no significant association between the expression levels of several genes, such as CDKN1A (p21 ${ }^{\text {Cipl }}$ ), VEGF, HIF1, HGF, CD133, SOX2,
OCT4, BAX and BCL2 in primary cancer cells and CRT effect on LPLN-positive status (data not shown); however, the mRNA levels of CXCL12 and CXCR4 in RC following CRT were $0.253 \pm 0.174$ (range, $0-9.03$ ) and $0.292 \pm 0.262$ (range, 0-13.86), respectively. Patients with consistently 
Table III. Patient and tumor characteristics according to enlarged LPLN after preoperative CRT.

\begin{tabular}{|c|c|c|c|}
\hline Characteristics & LPLN-positive & LPLN-negative & P-value \\
\hline Patients, $\mathrm{n}$ & 12 & 92 & \\
\hline Age (mean, years) & 60.8 & 62.6 & 0.58 \\
\hline Gender (male/female) & $10 / 2$ & $67 / 25$ & 0.43 \\
\hline Tumor distance (mean, $\mathrm{cm}$ ) & 3.7 & 4.0 & 0.71 \\
\hline \multicolumn{4}{|l|}{ Clinical UICC stage } \\
\hline I/II/III/IV & $0 / 0 / 12 / 0$ & $11 / 13 / 67 / 1$ & 0.23 \\
\hline cT4 $(\%)$ & $8(66.7)$ & $13(14.1)$ & $<0.0001$ \\
\hline $\mathrm{cN}$ metastasis $(\%)$ & $12(100)$ & $68(73.9)$ & 0.04 \\
\hline Histological differentiation & & & 0.47 \\
\hline High/moderate & 10 & 83 & \\
\hline Mucinous/poor & 2 & 9 & \\
\hline Pretreatment CEA (ng/ $\mu \mathrm{l})$ & 96.3 & 13.1 & $<0.0001$ \\
\hline \multicolumn{4}{|l|}{ CRT schedule } \\
\hline Short-course/long-course & $5 / 7$ & $52 / 40$ & 0.33 \\
\hline \multicolumn{4}{|l|}{ Adjuvant chemotherapy } \\
\hline Yes $(\%)$ & $11(91.2)$ & $85(92.4)$ & 1.00 \\
\hline \multicolumn{4}{|l|}{ Surgery } \\
\hline Yes $(\%)$ & $11(91.2)$ & $92(100)$ & 0.105 \\
\hline Surgical procedure & & & 0.0001 \\
\hline LAR & 0 & 28 & \\
\hline CAA & 4 & 58 & \\
\hline APR & 5 & 3 & \\
\hline Hartmann's procedure & 2 & 0 & \\
\hline Local excision & 0 & 3 & \\
\hline Intersphincter resection $(\%)$ & $4(33.3)$ & $39(42.4)$ & 0.55 \\
\hline APR $(\%)$ & $4(33.3)$ & $3(3.3)$ & $<0.0001$ \\
\hline \multicolumn{4}{|l|}{ Pathological UICC stage } \\
\hline 0/I/II/III/IV & $0 / 0 / 5 / 7 / 0$ & $7 / 24 / 31 / 28 / 2$ & 0.14 \\
\hline pN metastasis $(\%)$ & $7(58.3)$ & $28(30.4)$ & 0.054 \\
\hline CRM positivity (\%) & $1(9.0)$ & $2(2.2)$ & 0.20 \\
\hline \multicolumn{4}{|l|}{ Pathological effects } \\
\hline Grade $0 / 1 \mathrm{a} / 1 \mathrm{~b} / 2 / 3$ & $0 / 0 / 5 / 7 / 0$ & $7 / 24 / 31 / 28 / 2$ & 0.08 \\
\hline Grade $\geq 2(\%)$ & $4(36.4)$ & $34(37.0)$ & 0.81 \\
\hline $\mathrm{pCR}(\%)$ & $1(5.2)$ & $6(7.0)$ & 0.78 \\
\hline Mesorectal LN harvest & 11.8 & 8.1 & 0.051 \\
\hline Positive LNs (mean) & 3.7 & 0.7 & $<0.0001$ \\
\hline Negative LNs (mean) & 7.7 & 7.4 & 0.85 \\
\hline LN ratio (postitive/harvested) & 0.27 & 0.1 & 0.03 \\
\hline $\begin{array}{l}\text { Interval between CRT and surgery } \\
\text { (days), mean }\end{array}$ & 45.0 & 34.5 & 0.34 \\
\hline
\end{tabular}

Bold print indicates statistical significance. LPLN, lateral pelvic lymph nodes; UICC, Union for International Cancer Control; CRM, circumferential resection margin; CEA, carcinoembryonic antigen; CRT, chemoradiotherapy; LAR, low anterior resection; CAA, colo-anal anastomosis; APR, abdominoperineal rectal resection; pCR, pathological complete response.

positive LPLN status after CRT exhibited a significantly higher expression of the CXCL12 protein in cancer cells $(\mathrm{P}=0.02)$ compared with patients with a LPLN-negative status after CRT (Fig. 3).
Immunohistochemistry for CXCL12 in primary cancer, MLN and LPLN metastasis following CRT. CXCL12 staining was confirmed in 1 patient from this study, who developed local LPLN recurrence at 58.7 months after surgery. Specifically, 
Table IV. Univariate and multivariate analyses of factors considered to affect cancer-specific and relapse-free survival among patients with rectal cancer who received preoperative CRT.

\begin{tabular}{|c|c|c|c|c|c|c|}
\hline \multirow[b]{2}{*}{ Variables } & \multicolumn{3}{|c|}{ Univariate analysis } & \multicolumn{3}{|c|}{ Multivariate analysis } \\
\hline & OR & $95 \% \mathrm{CI}$ & P-value & OR & $95 \% \mathrm{CI}$ & P-value \\
\hline \multicolumn{7}{|l|}{ CSS } \\
\hline Age (64> vs. <64 years) & 1.20 & $0.47-3.01$ & 0.71 & - & - & - \\
\hline CRM positivity (yes vs. no) & 2.63 & $0.34-20.44$ & 0.36 & - & - & - \\
\hline AV $(<5$ vs. $5 \geq \mathrm{cm})$ & 3.571 & $0.82-15.63$ & 0.09 & - & - & - \\
\hline cT4 & 4.39 & $1.72-11.24$ & 0.002 & 3.65 & $1.28-10.42$ & 0.02 \\
\hline LPLN-positive before CRT & 2.03 & $0.76-5.41$ & 0.16 & - & - & - \\
\hline LPLN-positive after CRT & 3.75 & $1.40-10.00$ & 0.008 & 1.73 & $0.60-4.98$ & 0.31 \\
\hline pMLN metastasis & 3.45 & $1.35-8.85$ & 0.001 & 3.72 & $1.34-10.31$ & 0.01 \\
\hline Pathological effect (grade 0-1 vs. 2-3) & 1.41 & $0.53-3.77$ & 0.49 & - & - & - \\
\hline CEA level (6> vs. $<6$ ng $/ \mu \mathrm{l})$ & 5.35 & $1.53-18.89$ & 0.009 & 3.20 & $0.86-11.90$ & 0.08 \\
\hline \multicolumn{7}{|l|}{ RFS } \\
\hline Age $(64>$ vs. $<64$ years $)$ & 1.39 & $0.64-3.13$ & 0.40 & - & - & - \\
\hline CRM positivity (yes vs. no) & 4.86 & $1.12-21.12$ & 0.03 & 4.55 & $0.87-23.76$ & 0.07 \\
\hline AV (<5 vs. $5 \geq \mathrm{cm})$ & 1.59 & $0.60-3.76$ & 0.38 & - & - & - \\
\hline cT4 & 2.53 & $1.11-5.75$ & 0.03 & 2.17 & $0.87-5.32$ & 0.10 \\
\hline LPLN-positive before CRT & 1.33 & $0.53-3.32$ & 0.54 & - & - & - \\
\hline LPLN-positive after CRT & 2.46 & $0.98-6.17$ & 0.05 & 1.48 & $0.57-3.85$ & 0.42 \\
\hline pMLN metastasis & 4.20 & $1.89-9.35$ & 0.0004 & 4.61 & $2.00-10.53$ & 0.0003 \\
\hline Pathological effect (grade 0-1 vs. 2-3) & 1.91 & $0.80-4.5$ & 0.14 & - & - & - \\
\hline CEA level (6> vs. <6 ng/ $\mu \mathrm{l})$ & 2.20 & $0.97-5.00$ & 0.06 & - & - & - \\
\hline
\end{tabular}

Bold print indicates statistical significance. OR, odds ratio; CI, confidence interval; CSS, cancer-specific survival; CRM, circumferential resection margin; AV, tumor distance from anal verge; LPLN, lateral pelvic lymph node; pMLN, pathological mesorectal lymph node metastasis; CEA, carcinoembryonic antigen; RFS, relapse-free survival.
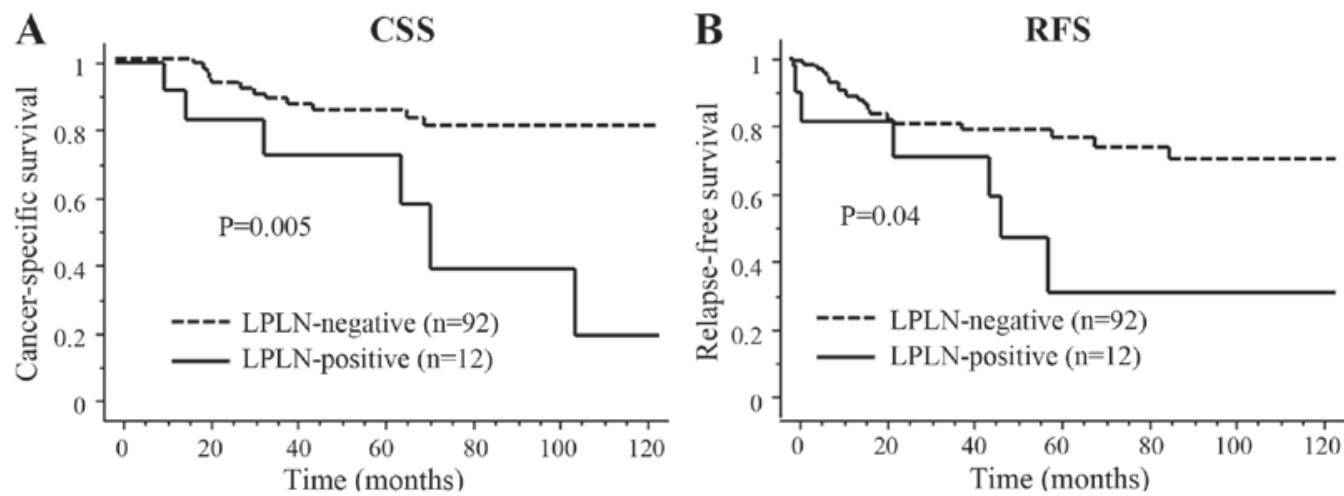

Figure 1. Kaplan-Meier survival estimates for (A) cancer-specific survival (CSS) and (B) relapse-free survival (RFS) in the LPLN-positive and LPLN-negative groups after preoperative CRT. LPLN, lateral pelvic lymph nodes; CRT, chemoradiotherapy.

immunohistochemical staining for the CXCL12 protein was observed in the cytoplasm of cancer cells in the primary tumor, MLN metastasis and LPLN metastasis specimens harvested for staging biopsy (Fig. 4).

\section{Discussion}

The incidence of LPLN-positive status (18.3\%) in our study was similar to that of pathological LPLN metastasis of previous reports, in which patients underwent surgery alone (1-5). Following preoperative CRT, 7 of the 19 (36.8\%) initially LPLN-positive patients exhibited LPLN downsizing to $<7 \mathrm{~mm}$; none of these 7 patients developed recurrence. The remaining 12 patients who remained LPLN-positive after preoperative CRT exhibited significantly poorer CSS and RFS compared with the LPLN-negative after CRT group. Previously reported 5-year survival rates of patients who underwent TME with LPLN dissection in the presence of LPLN metastases 

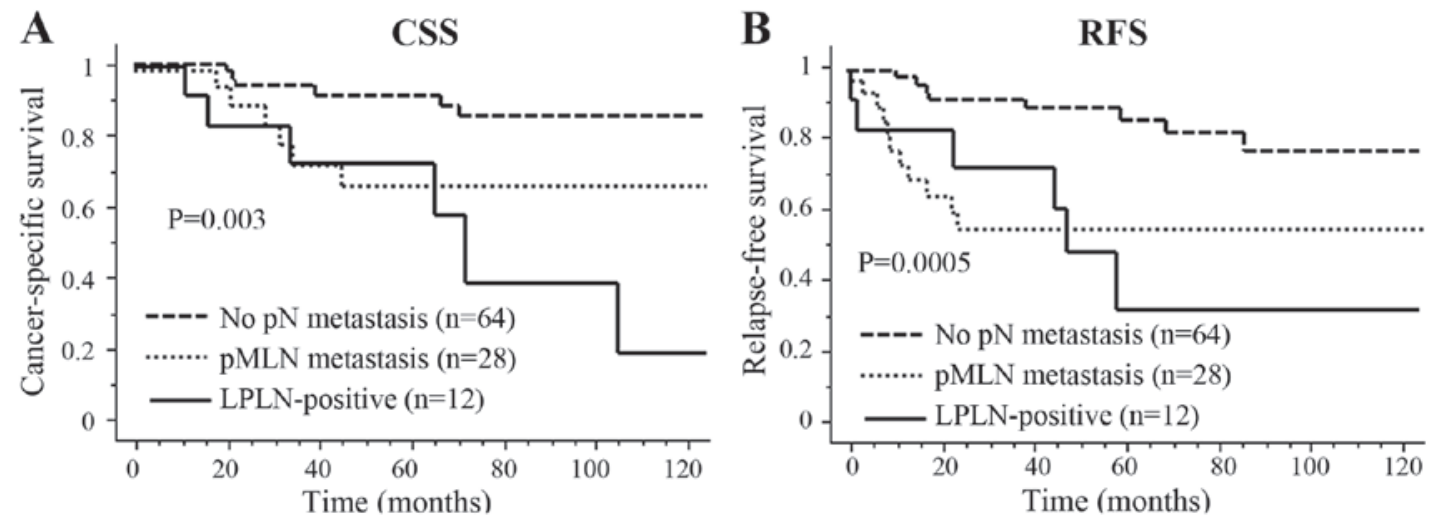

Figure 2. Kaplan-Meier survival analysis showed significant differences in (A) cancer-specific survival (CSS) and (B) relapse-free survival (RFS) among three groups, namely the pNO, pMLN metastasis and LPLN-positive groups, after CRT. MLN, mesorectal lymph nodes; LPLN, lateral pelvic lymph nodes; CRT, chemoradiotherapy.
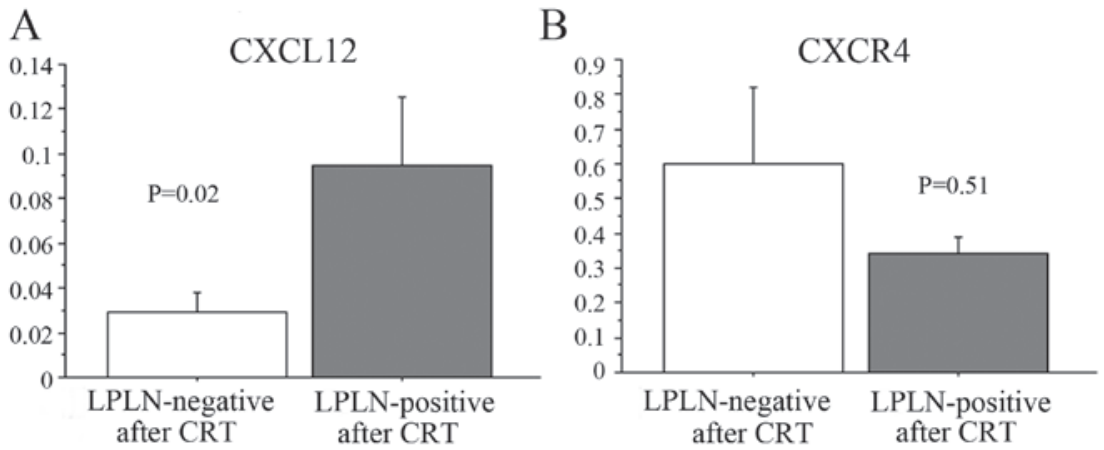

Figure 3. Differences in gene expression patterns of CXCL12 (A) and CXCR4 (B) in primary cancer cells according to the response of LPLN-positive status to CRT. CXCL12, C-X-C motif chemokine 12; CXCR4, C-X-C chemokine receptor type 4; LPLN, lateral pelvic lymph nodes; CRT, chemoradiotherapy.
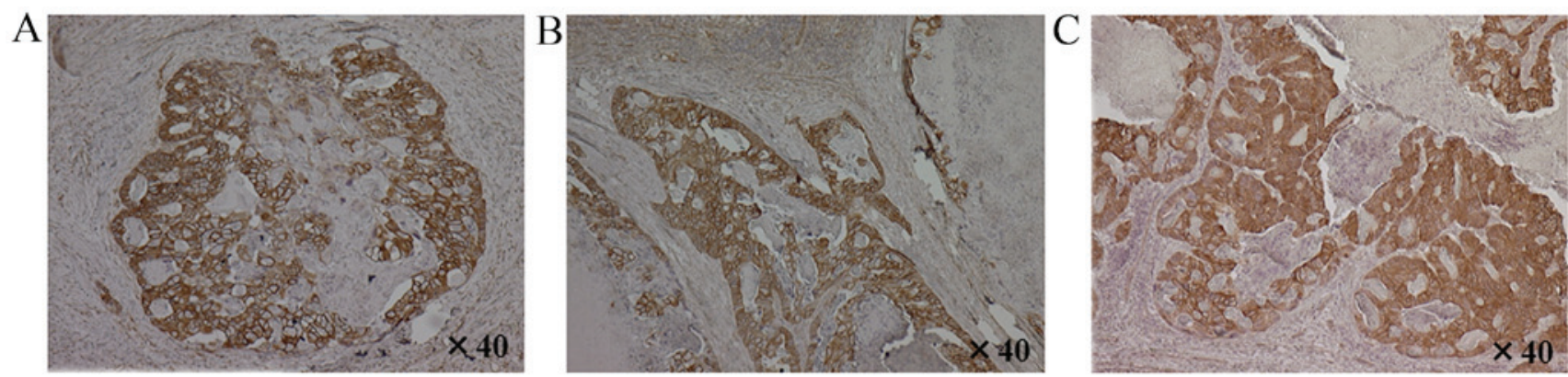

Figure 4. Immunohistochemical staining for CXCL12 was observed in the cytoplasm of cancer cells in (A) primary cancer, (B) mesorectal lymph node metastasis and (C) lateral pelvic lymph node metastasis in 1 patient from the present study.

are $\sim 45 \%(2,3,16,17)$, which are inferior to that of our patients with enlarged LPLN after CRT who had TME alone. Although the survival rates of the present study cannot be compared to previously reported rates, a proportion of patients with LPLN metastasis may clearly be treated by CRT-TME alone. However, locally advanced RC with extended LPLN metastasis may not be associated with a good prognosis, even after preoperative CRT or LPLN dissection alone. Therefore, Western investigations have focused on the multidisciplinary management of patients with RC beyond TME planes (18). The National Comprehensive Cancer Network guidelines currently recommend that clinically suspicious nodes beyond the fields of resection should be biopsied or removed if possible (19).
A recent Japanese study demonstrated that $66 \%$ of patients undergoing preoperative CRT followed by LPLN dissection had pathological LPLN metastasis, with favorable 3-year local recurrence rates of $7.1 \%$ in the TME group and $2.7 \%$ in the LPLN dissection group (9). By contrast, Quadros et al reported that metastases to the LPLN indicated unfavorable survival at 50 months (28.6\% for LPLN-positive vs. $84.5 \%$ for LPLN-negaitive cases) after preoperative CRT followed by LPLN dissection (20). Our study may help elucidate whether the survival rate of the irradiated patients with LPLN metastases would be worse if LPLN dissection was not performed. Our study used the morphological change of LPLN-positive status as a marker for prognosis of patients who underwent 
CRT-TME. We observed that the presence of clinically positive LPLN does not affect prognosis after TME alone when enlargement improves after CRT; however, the survival of patients who remained LPLN-positive after preoperative CRT was unsatisfactory.

In the present study, survival differences between pMLN metastasis and LPLN-positive status after CRT were confirmed $>5$ years after surgery, mainly due to delayed local recurrence. In addition, whether residual LPLN metastases promote the spread of cancer cells by amplifying their numbers after such a long time, thus serving as a launch pad for further systemic metastases, remains to be elucidated. As little is known on the underlying molecular mechanisms, several CRT-related genes in primary tumors were compared between LPLN-positive responders and non-responders to CRT. We confirmed that only CXCL12 expression in primary cancer cells was significantly higher in responders compared with that in non-responders, as determined by LPLN shrinkage following preoperative CRT. CXCL12 is a chemokine expressed by both cancer and stromal cells $(21,22)$. Reportedly, CXCL12 and its receptor, CXCR4, play important roles in local progression, dissemination and immune evasion of colorectal cancer cells (23-25). Our recent study also suggested that stromal CXCL12 and CXCR4 expression following preoperative CRT is associated with distant recurrence and poor prognosis in RC (15). Therefore, we hypothesized that our RC patients with positive LPLN after CRT may have oncogenetic potential for cancer progression, resulting in delayed recurrence; thus, more intensive multimodality therapies, pre- as well as postoperatively, may improve survival in the light of these molecular characteristics.

The present study had certain limitations: It was a retrospective study with relatively few patients, and positive LPLN do not necessarily harbor metastases. However, to the best of our knowledge, the present study was the first to investigate the effect of preoperative CRT, including genetic potential, on the LPLN-positive status of patients not undergoing LPLN dissection.

In conclusion, enlarged LPLN identified on pretreatment imaging do not affect CSS or RFS after CRT-TME, except in cases that remain LPLN-positive after preoperative CRT. LPLN downsizing to $<7 \mathrm{~mm}$, as determined radiographically, may be a marker for prognosis of patients with $\mathrm{RC}$ who undergo CRT-TME, whereas LPLN dissection may be unnecessary when all LPLN become negative $(<7 \mathrm{~mm})$ after CRT. Strategies to improve survival in patients with LPLN-positive status after CRT may include selective LPLN dissection or more aggressive multimodality therapies.

\section{References}

1. Ueno M, Oya M, Azekura K, Yamaguchi T and Muto T: Incidence and prognostic significance of lateral lymph node metastasis in patients with advanced low rectal cancer. Br J Surg 92: 756-763, 2005.

2. Sugihara K, Kobayashi H, Kato T, Mori T, Mochizuki H, Kameoka S, Shirouzu K and Muto T: Indication and benefit of pelvic sidewall dissection for rectal cancer. Dis Colon Rectum 49: 1663-1672, 2006.

3. Ueno H, Mochizuki H, Hashiguchi Y, Ishiguro M, Miyoshi M, Kajiwara Y, Sato T, Shimazaki H and Hase K: Potential prognostic benefit of lateral pelvic node dissection for rectal cancer located below the peritoneal reflection. Ann Surg 245: 80-87, 2007.
4. Kobayashi H, Mochizuki H, Kato T, Mori T, Kameoka S, Shirouzu K and Sugihara K: Outcomes of surgery alone for lower rectal cancer with and without pelvic sidewall dissection. Dis Colon Rectum 52: 567-576, 2009.

5. Akiyoshi T, Watanabe T, Miyata S, Kotake K, Muto T and Sugihara K; Japanese Society for Cancer of the Colon and Rectum: Results of a Japanese nationwide multi-institutional study on lateral pelvic lymph node metastasis in low rectal cancer: Is it regional or distant disease? Ann Surg 255: 1129-1134, 2012.

6. Watanabe T, Tsurita G, Muto T, Sawada T, Sunouchi K, Higuchi Y, Komuro Y, Kanazawa T, Iijima T, Miyaki M and Nagawa H: Extended lymphadenectomy and preoperative radiotherapy for lower rectal cancers. Surgery 132: 27-33, 2002.

7. Yano H, Saito Y, Takeshita E, Miyake O and Ishizuka N: Prediction of lateral pelvic node involvement in low rectal cancer by conventional computed tomography. Br J Surg 94: 1014-1019, 2007.

8. Dharmarajan S, Shuai D, Fajardo AD, Birnbaum EH, Hunt SR, Mutch MG, Fleshman JW and Lin AY: Clinically enlarged lateral pelvic lymph nodes do not influence prognosis after neoadjuvant therapy and TME in stage III rectal cancer. J Gastrointest Surg 15: 1368-1374, 2011.

9. Akiyoshi T, Ueno M, Matsueda K, Konishi T, Fujimoto Y, Nagayama S, Fukunaga Y, Unno T, Kano A, Kuroyanagi H, et al: Selective lateral pelvic lymph node dissection in patients with advanced low rectal cancer treated with preoperative chemoradiotherapy based on pretreatment imaging. Ann Surg Oncol 21: 189-196, 2014.

10. Lim SB, Yu CS, Kim CW, Yoon YS, Park SH, Kim TW, Kim JH and Kim JC: Clinical implication of additional selective lateral lymph node excision in patients with locally advanced rectal cancer who underwent preoperative chemoradiotherapy. Int J Colorectal Dis 28: 1667-1674, 2013.

11. Japanese Society for Cancer of the Colon and Rectum: Japanese classification of colorectal carcinoma. 2nd English edition. Kanehara \& Co. Ltd., Tokyo, pp7-10, 2009.

12. Yoshikawa R, Kusunoki M, Yanagi H, Noda M, Furuyama JI, Yamamura T and Hashimoto-Tamaoki T: Dual antitumor effects of 5-fluorouracil on the cell cycle in colorectal carcinoma cells: A novel target mechanism concept for pharmacokinetic modulating chemotherapy. Cancer Res 61: 1029-1037, 2001.

13. Inoue $Y$, Okigami M, Kawamoto A, Okugawa Y,Hiro J, Saigusa S, Toiyama Y, Tanaka K, Mohri Y and Kusunoki M: Phase I study of 5-fluorouracil, leucovorin and bevacizumab in combination with radiation therapy in patients with locally advanced rectal cancer. Mol Clin Oncol 1: 511-516, 2013.

14. Saigusa S, Tanaka K, Toiyama Y, Matsushita K, Kawamura M, Okugawa Y, Hiro J, Inoue Y, Uchida K, Mohri Y and Kusunoki M: Gene expression profiles of tumor regression grade in locally advanced rectal cancer after neoadjuvant chemoradiotherapy. Oncol Rep 28: 855-861, 2012.

15. Saigusa S, Toiyama Y, Tanaka K, Yokoe T, Okugawa $Y$, Kawamoto A, Yasuda H, Inoue Y, Miki C and Kusunoki M: Stromal CXCR4 and CXCL12 expression is associated with distant recurrence and poor prognosis in rectal cancer after chemoradiotherapy. Ann Surg Oncol 17: 2051-2058, 2010.

16. Moriya Y, Sugihara K, Akasu T and Fujita S: Importance of extende lymphadenectomy with lateral node dissection for advanced lower rectal cancer. World J Surg 21: 728-732, 1997.

17. Sugihara K, Moriya Y, Akasu T and Fujita S: Pelvic autonomic nerve preservation for patients with rectal carcinoma. Oncologic and functional outcome. Cancer 78: 1871-1880, 1996.

18. Beyond TME Collaborative: Consensus statement on the multidisciplinary management of patients with recurrent and primary rectal cancer beyond total mesorectal excision planes. Br J Surg 100: 1009-1014, 2013.

19. Benson AB III, Bekaii-Saab T, Chan E, Chen YJ, Choti MA, Cooper HS, Engstrom PF, Enzinger PC, Fakih MG, Fuchs CS, et al: Rectal cancer. J Natl Compr Canc Netw 10: 1528-1564, 2012.

20. Quadros CA, Falcão MF, Carvalho ME and Ladeia PA: Metastases to retroperitoneal or lateral pelvic lymph nodes indicated unfavorable survival and high pelvic recurrence rates in a cohort of 102 patients with low rectal adenocarcinoma. J Surg Oncol 106: 653-658, 2012.

21. Salvucci O, Yao L, Villalba S, Sajewicz A, Pittaluga S and Tosato G: Regulation of endothelial cell branching morphogenesis by endogenous chemokine stromal-derived factor-1. Blood 99: 2703-2711, 2001. 
22. Müller A, Homey B, Soto H, Ge N, Catron D, Buchanan ME, McClanahan T, Murphy E, Yuan W, Wagner SN, et al: Involvement of chemokine receptors in breast cancer metastasis. Nature 410: 50-56, 2001.

23. Kim J, Takeuchi H, Lam ST, Turner RR, Wang HJ, Kuo C, Foshag L, Bilchik AJ and Hoon DS: Chemokine receptor CXCR4 expression in colorectal cancer patients increases the risk for recurrence and for poor survival. J Clin Oncol 23: 2744-2753, 2005.

24. Matsusue R, Kubo H, Hisamori S, Okoshi K, Takagi H, Hida K, Nakano K, Itami A, Kawada K, Nagayama S and Sakai Y: Hepatic stellate cells promote liver metastasis of colon cancer cells by the action of SDF-1/CXCR4 axis. Ann Surg Oncol 16: 2645-2653, 2009.
25. Ottaiano A,Franco R,Aiello Talamanca A,Liguori G, TatangeloF Delrio P, Nasti G, Barletta E, Facchini G, Daniele B, et al: Overexpression of both CXC chemokine receptor 4 and vascular endothelial growth factor proteins predicts early distant relapse in stage II-III colorectal cancer patients. Clin Cancer Res 12: 2795-2803, 2006. 